Original Paper

http://indexmedicus.afro.who.int

\title{
Caractérisation des sols de la vallée rizicole de Tamra dans l’île de Mar, Centre-Ouest du Sénégal
}

\author{
Jean Henri Bienvenue SENE ${ }^{1}$, François MATTY ${ }^{1^{*}}$ et Malaïny DIATTA ${ }^{2}$ \\ ${ }^{1}$ Laboratoire de Science du Sol, Institut des Sciences de l'Environnement, Faculté des Sciences et Techniques. \\ Université Cheikh Anta Diop, BP : 5005 Dakar-Fann, Sénégal. \\ ${ }^{2}$ Centre National de Recherches Forestières, Institut Sénégalais de Recherches Agricoles, BP 2312 Dakar- \\ Hann, Sénégal. \\ *Auteur correspondant, E-mail : gosmatty@yahoo.fr; Tél : +221 774020918
}

\section{RESUME}

Tamra est une vallée très sollicitée pour la culture du riz par les populations des quatre villages implantés dans l'île de Mar. L'étude pédologique a été menée suivant trois toposéquences transversales le long de la vallée. Les différents types de sols rencontrés dans ce milieu fluvio-marin sont disposés d'une manière quasi-concentrique autour de la vallée. Les sols sont affectés par le processus de salinisation et d'acidification. Les causes principales de l'avancée de ce biseau salé le long de la vallée seraient liées à l'environnement climatique peu favorable qui sévit depuis plusieurs années et qui s'exprime par des sécheresses récurrentes. La texture sableuse de ces sols, à dominance de sables fins, et leur niveau topographique font que certains d'entre eux sont exceptionnellement inondés. Ainsi, la réalisation d'une carte d'aptitude culturale des sols s'impose pour une meilleure utilisation des terres de la vallée de Tamra.

() 2014 International Formulae Group. All rights reserved.

Mots clés : Tamra, sols, fertilité, salinisation, acidification.

\section{INTRODUCTION}

La baisse pluviométrie ces quarante dernières années en zone sahélienne a beaucoup affecté l'environnement biophysique sur le littoral du Sénégal. Ainsi le bassin versant du SineSaloum est soumis à divers phénomènes de dégradation des terres dont la salinisation et l'acidification (Sadio, 1991). Ces phénomènes auraient pour origine les longues et fréquentes périodes de sécheresse et l'exploitation abusive du couvert végétal par l'homme. Les conséquences environnementales sont désastreuses car le biseau salé a considérablement avancé dans les principaux cours d'eau du Sénégal (Marius, 1985), contaminant ainsi les sols cultivables. Il s'en est suivi alors la baisse de la productivité des sols, la décadence du couvert végétal notamment la mangrove, la rareté de la faune sauvage et l'abandon par les populations des terres réservées à la culture du riz (Sadio, 1991). Les populations s'adonnent actuellement à la saliculture sur ces mêmes terres dégradées chimiquement. Devant l'ampleur de ce phénomène de dégradation de l'environnement, des tentatives de récupération des terres affectées ont été mises en œuvre. Il s'agit essentiellement de l'érection de digues et barrages anti-sel au 
niveau des affluents, du reboisement des terres salées avec des essences forestières halotolérantes et de l'amendement des sols par le phosphogypse. C'est ainsi que dans la vallée rizicole de Tamra, située dans l'île de Mar, ont été mises en place une digue anti-sel en aval et une digue de retenue des eaux de pluies en amont pour une restauration agronomique des sols. Quatre ans après la réalisation de ces aménagements hydroagricoles, une étude diagnostique a été menée dans la vallée en l'absence de données sur la situation de référence, en l'occurrence avant la mise en place des digues. L'objectif de ce travail est de faire l'inventaire des différents types de sols et de les caractériser dans la perspective de leur utilisation rationnelle sur la base de leurs potentialités. Ce travail scientifique peut servir de situation de référence aux études ultérieures sur le suivi de l'évolution des sols de la vallée rizicole de Tamra.

\section{MATERIEL ET METHODES Le site d'étude}

L'île de Mar sur le plan administratif est située dans la région de Fatick au Sénégal. Le climat est caractérisé par une pluviométrie très irrégulière qui connaît depuis quelques décennies un déficit généralisé. Le site d'étude se trouve sur l'isohyète $700 \mathrm{~mm}$. Les températures sont très élevées et les variations thermiques entre les maxima et les minima annuels sont importantes. En effet, $40{ }^{\circ} \mathrm{C}$ sont enregistrés en avril/mai et $15{ }^{\circ} \mathrm{C}$ en janvier/février. Du fait des températures élevées, l'évaporation est aussi très importante pendant les mois de mars, avril et mai où elle atteint $310 \mathrm{~mm}$ (Evaporation Bac) au niveau de la station régionale de Fatick (Marius, 1979). Elle favorise la salinisation par remontée capillaire. L'humidité relative est un facteur tout aussi déterminant dans les phénomènes pédogénétiques que la pluviométrie, les températures et l'évaporation. Elle augmente à partir d'avril/mai jusqu'en août/septembre et subit une baisse à partir du mois d'octobre. Ce régime unimodal est dû à l'existence d'une seule saison des pluies au cours de l'année. L'humidité relative permet la mise en place sur le sol d'une croûte efficace contre les effets de la déflation éolienne. Cette humidité relative est toujours élevée au cours des minima de températures (fin de la nuit) et contribue à atténuer l'effet des périodes de sécheresse (Diop, 1986). On note une grande diversité des sols et leurs caractéristiques morphologiques et chimiques semblent être étroitement liées à leur position topographique et à la dynamique du réseau hydrographique (Sadio, 1991). Ainsi, à chaque unité géomorphologique semble correspondre un type de sol et une végétation caractéristiques. La vallée de Tamra est longue de $2,7 \mathrm{Km}$; son périmètre est de $7,1 \mathrm{~km}$ et sa superficie de 156 ha. Cette vallée joue le rôle d'affluent du marigot salé de Djilor. L'écoulement des eaux dans cette vallée se fait dans la direction SO-NE. Notons que Mar fait partie du chapelet d'îles enfermées dans le vaste réseau hydrographique du bassin versant du SineSaloum (Figure 1).

Ce réseau est constitué de trois bras de mer que sont le Bandiala au Sud, le Diomboss au centre et le Saloum au Nord. De nos jours, du fait d'un déficit drastique en eaux de pluies longtemps observé et de la pente trop faible de ce domaine fluvio-marin, le biseau salé a envahi toutes les ramifications du réseau hydrographique qui, désormais, fonctionne comme un réseau d'irrigation en eau salée contaminant les sols et les nappes phréatiques (Marius, 1985 ; Diarra, 1999). Les sols de la vallée de Tamra méritent une attention particulière vu la position insulaire du terroir de Mar et le phénomène croissant de salinisation des terres dans les îles du Saloum d'une manière générale.

\section{La prospection pédologique}

Les travaux de prospection ont nécessité tout d'abord la subdivision de la vallée de Tamra en trois grandes unités géomorpho-pédologiques suivant sa longueur. C'est ainsi qu'ont été délimitées l'unité de la basse vallée, celle de la moyenne vallée et celle de la haute vallée (Figure 2). La caractérisation des sols a été réalisée suivant trois toposéquences transversales représentatives. La première toposéquence est située en aval et compte 6 profils, la deuxième se trouve en moyenne vallée avec 6 profils et la troisième en haute vallée avec 5 profils. Un profil a été creusé vers l'extrémité amont, ce qui fait un total de dix huit profils 
pédologiques qui ont fait l'objet d'une description complète. Quatorze échantillons de sol ont été prélevés dans les profils typiques pour des analyses physicochimiques. Ces échantillons ont été d'abord séchés à l'air libre avant d'être soumis à l'analyse.

\section{Les analyses de sols et de nappes}

L'accent est mis sur les caractéristiques physiques et chimiques des sols. Ainsi les principaux paramètres déterminés sont: la granulométrie, la conductivité électrique (indice de salinité), le $\mathrm{pH}$, le carbone organique (selon la méthode modifiée de Walkley-Black, 1965), l'azote total (selon Kjeldahl), le phosphore assimilable (selon Bray I, 1945), la capacité d'échange cationique, le bilan ionique de la solution filtrée de l'extrait aqueux 1/5 (Baize, 1988). La détermination du bilan ionique dans cet extrait aqueux a permis de dresser le profil salin du sol en moyenne et haute vallée et ainsi d'avoir une idée sur la dynamique des sels suivant la profondeur. Notons que les analyses ont été effectuées au laboratoire de pédologie du Centre de recherche de l'Institut Sénégalais de Recherches Agricoles (ISRA) à Bambey.

\section{Le traitement des données}

Les données obtenues au laboratoire ont été confrontées à celles morphologiques relevées sur le terrain. Ceci a permis de classer les différentes unités de sols rencontrées. Le système de classification des sols utilisé est celui relevant du référentiel pédologique français de 1967 (CPCS, 1967). La carte de présentation de la vallée a été réalisée à l'aide des logiciels Excel, Surfer 8, ArcGIS 9. Les données de base pour la réalisation de cette carte sont constituées des coordonnées géographiques du contour de la vallée, des coordonnées des 18 profils pédologiques creusés et de celles des deux digues.
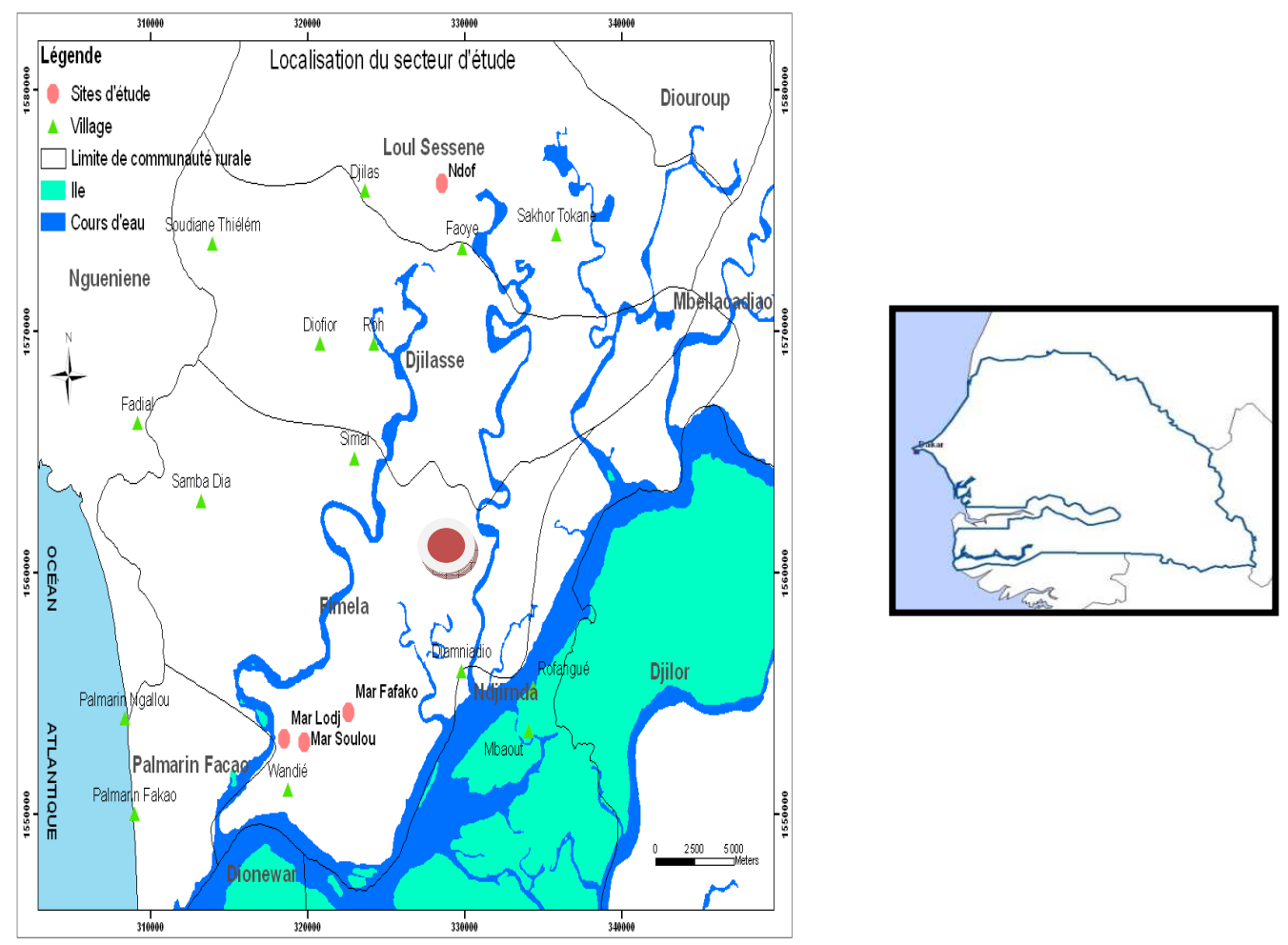

Figure 1: Carte de situation de l'île de Mar. 


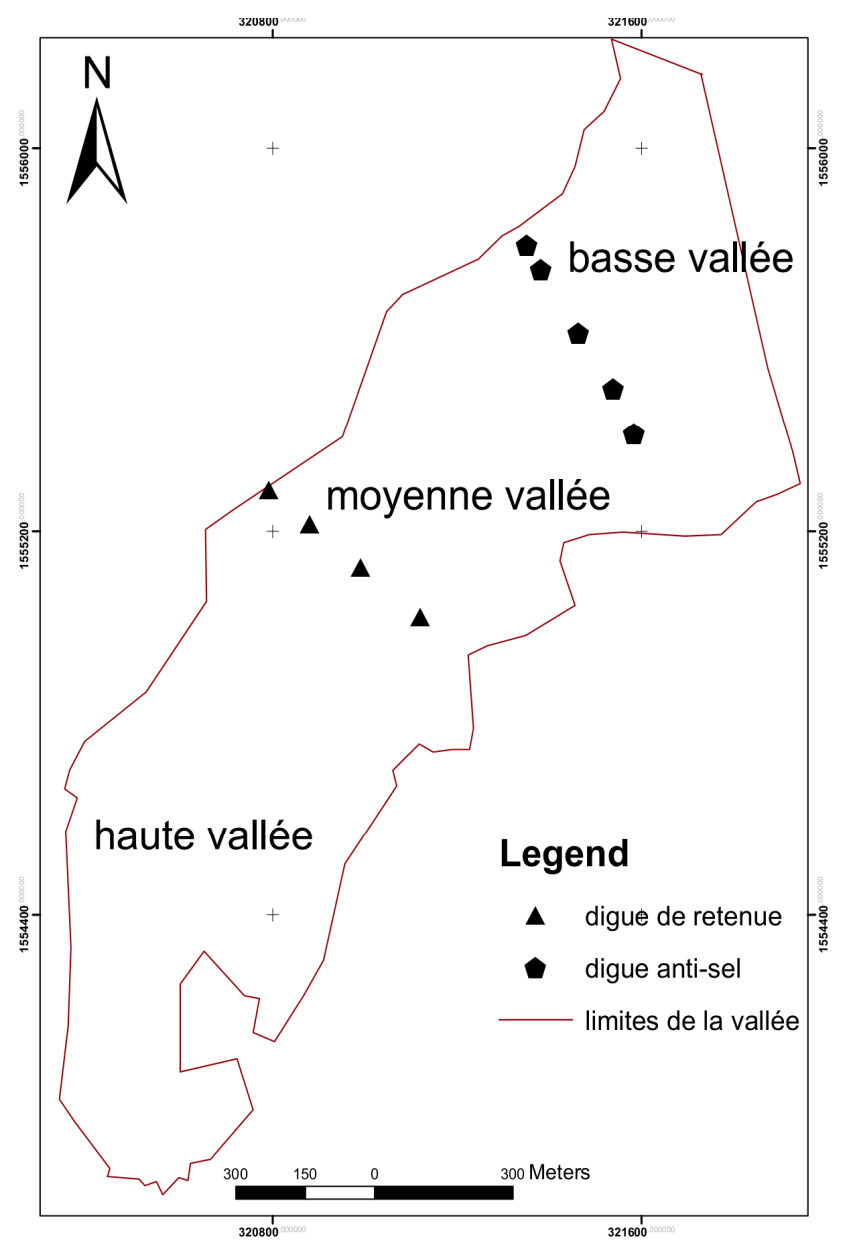

Figure 2 : Présentation de la vallée de Tamra (à l'aide d'ARC GIS 9).

\section{RESULTATS}

\section{Les facteurs de pédogenèse}

Le bassin du Sine-Saloum a connu durant le quaternaire récent des fluctuations climatiques (transgression et régression) ayant influencé les dépôts de sédiments en quantité et en qualité notamment dans les anciens golfes marins (Barusseau et al., 1985). Ainsi l'analyse granulométrique effectuée par (Marius, 1979) dans différentes îles du Saloum a révélé des sédiments essentiellement sableux (sables fins) et argileux. Au plan minéralogique, on y trouve du quartz qui aurait pour origine les sables du Continental Terminal. Les principaux minéraux argileux rencontrés sont la kaolinite, la montmorillonite, la smectite et l'illite. Ce cortège minéralogique est souvent associé à la pyrite $\left(\mathrm{FeS}_{2}\right)$, roche amorphe responsable de l'apparition, sous certaines conditions, des sols à caractère salé, sulfaté acide. C'est dans ce milieu complexe, riche en sables marins fins, en minéraux argileux diversifiés, comportant de la mangrove reposant sur de la vase que se sont formés les différents types de sols rencontrés dans la vallée de Tamra.

Ce travail a permis d'identifier le long de la vallée de Tamra quatre principales unités de sols : 
- sol hydromorphe minéral, salé, sulfaté acide mature, sableux (unité de sol 1 ou US 1);

- sol hydromorphe minéral, salé, sableux (unité de sol 2 ou US 2);

- sol peu évolué, d'apport hydromorphe, sableux (unité de sol 3 ou US 3) ;

- sol ferrugineux tropical peu lessivé, hydromorphe, sableux (unité de sol 4 ou US 4).

\section{Caractéristiques morpho-analytiques des} sols

Unité de sol 1: Sol hydromorphe minéral, salé, sulfaté acide mature

Ce sol est classé hydromorphe minéral, salé, sulfaté acide mature, sableux (US 1). Il n'a été identifié que dans la basse vallée, le long du chenal principal d'écoulement (lit mineur). C'est un sol qui subit une saturation quasi permanente en eaux (douces ou salées). Il est presque dénudé (désert salé) et parsemé de quelques buttes de sables recouvertes essentiellement d'une végétation herbacée constituée de Cyperaceae et de quelques pieds de Spermacoce verticillata. Le matériau d'ensemble est sableux et vaseux en profondeur, avec une nette prédominance des sables fins. La vase est noire et instable (boulant). Il a été noté une forte fréquence de taches ocre jaune verdâtre et ocre jaune en semi-profondeur et en profondeur alors que le matériau est bariolé ocre rougeâtre et jaunâtre à $90 \%$ dans les horizons superficiels. Quelques concrétions ferrugineuses friables («irons pipes») ont été observées dans les horizons de surface. Elles sont assez nombreuses en semi-profondeur. Des racines pourries de Tamarix senegalensis ont aussi été identifiées en profondeur. Ce sol est extrêmement salé $(\mathrm{CE}=12,421 \mathrm{mS} / \mathrm{cm})$ et hyper acide $(\mathrm{pH}=2,4)$ (Annexe). Il présente cependant un bon drainage latéral (hypodermique) mais le drainage vertical est jugé mauvais.
Unité de sol 2 : Sol hydromorphe minéral, salé

Ce type de sol est présent au niveau des terrasses moyenne et basse, donc dans une zone inondable. En basse vallée, il donne l'image d'un désert salé avec des efflorescences salines. Il est parsemé de buttes sableuses. Dans la moyenne vallée, il est recouvert d'un tapis herbacé dominé par Spermacoce verticillata, Hygrophila auriculata et Ipomea asarifolia. On note la présence de quelques pieds d'Acacia seyal et d'un Acacia nilotica au centre de la moyenne vallée. C'est un sol profond, caractérisé par un épais horizon rougeâtre dans la première moitié de son profil, surmonté d'un horizon humifère relativement épais reposant sur un matériau de profondeur sableux et vaseux, de couleur noire et instable (boulant). Par endroit, ce sol présente, au milieu du profil, un passage sableux blanchâtre et boulant. La présence de cette fine couche particulière indique que ce sol bénéficie d'un bon drainage hypodermique latéral. La texture d'ensemble est sableuse avec une nette prédominance des sables fins.

Ce type de sol a une teneur relativement bonne en matière organique $(1,9 \%)$. Une matière organique qui se minéralise bien (rapport $\mathrm{C} / \mathrm{N}$ variant entre 8 et 12). Il est bien pourvu en azote $(0,89 \%$ ). On note cependant de faibles teneurs voire une carence en phosphore assimilable $\left(\mathrm{P}_{2} \mathrm{O}_{5}\right)$ et en potassium (K). Il y a aussi un déséquilibre, en proportions, entre les teneurs en potassium et en magnésium (Boyer, 1982). En effet, la teneur en $\mathrm{Mg}$ fait 8 fois celle du K (rapport $\mathrm{K} / \mathrm{Mg}$ égal à $1 / 8$ ). La nutrition minérale des cultures en $\mathrm{K}$ pourrait être menacée par l'excès relatif du $\mathrm{Mg}$.

La capacité d'échange cationique de cette unité de sol est très faible. Elle n'excède pas 1,6 meq/100 g. Le sol est saturé en bases échangeables notamment en Sodium $\left(\mathrm{Na}^{+}\right)$. Ainsi le taux de sodium échangeable (TSE) est supérieur à $15 \%$, ce qui rend le milieu relativement toxique (Baize, 1988). Le sol est très salé en basse vallée $(\mathrm{CE}=12,4 \mathrm{mS} / \mathrm{cm})$ et 
moyennement salé en moyenne vallée $(\mathrm{CE}=$ $0,85 \mathrm{mS} / \mathrm{cm}$ ). Il est aussi très acide (Annexe).

Unité de sol 3: Sol peu évolué d'apport, hydromorphe

Cette unité de sol se rencontre principalement sur la terrasse moyenne des berges de la vallée et parfois sur la terrasse basse (en haute vallée). Il est recouvert d'un dense tapis herbacé constitué essentiellement de Spermacoce verticillata et Ipomea asarifolia. Il est caractérisé par un épais horizon humifère (35 à $40 \mathrm{~cm}$ d'épaisseur). Il est bariolé ocre jaunâtre en surface et rouge vif dans la deuxième moitié de son profil. Cette coloration multiple rouge vif à rouge violet dénote une forte fluctuation de la nappe phréatique le long du profil. Le matériau d'ensemble est sableux avec une nette prédominance des sables fins. Les teneurs en matière organique sont faibles dans la moyenne vallée et assez bonne $(1,38 \%)$ dans la haute vallée. Ce sol est relativement bien pourvu en azote $(0,53 \%$ ) et pourtant la matière organique se minéralise lentement. Il est faiblement pourvu en phosphore assimilable et en potassium. On note également un excès de magnésium par rapport au potassium. Une carence en potassium pourrait par conséquent être accentuée par ce déséquilibre ionique. La capacité d'échange cationique (CEC) est jugée faible dans ce sol très sableux. C'est un sol moyennement saturé en bases dans la haute vallée $(\mathrm{V}=60,5 \%)$. Le taux de sodium échangeable (TSE) est respectivement de 44,05 et $18,49 \%$ dans la moyenne vallée et dans la haute vallée (Annexe). Il est très acide devenant hyper acide en profondeur. Il est jugé non salé mais présente des traces de sels dans les horizons de profondeur en moyenne vallée.

Unité de sol 4 : Sol ferrugineux tropical, peu lessivé, hydromorphe

Cette unité de sol est présente sur la terrasse haute (au niveau des berges de la vallée) et par endroit sur la terrasse moyenne comme en haute vallée.

Sur le plan hydrologique, ce sol se trouve dans des zones exceptionnellement inondables. Il est surmonté d'un couvert ligneux diversifié: Acacia ataxacantha, Borassus aethiopium, Maytenus senegalensis, Detarium senegalense, Combretum glutinosum, Sclerocarya birrea, Adansonia digitata. La strate herbacée est essentiellement constituée de Spermacoce verticillata. C'est un sol très profond avec un épais horizon humifère. Il est surtout caractérisé par la présence d'un horizon rouge violet avec de nombreuses taches ocre jaune verdâtre dans la première moitié de son profil. Ceci témoigne d'une forte fluctuation de la nappe phréatique confirmant le caractère hydromorphe de ce sol. Des concrétions ferrugineuses sont perceptibles au niveau de cet horizon diagnostic rouge violet. La texture d'ensemble est sableuse avec une nette prédominance des sables fins.

Cette unité de sol a un taux de matière organique très faible $(0,44 \%)$. Il est moyennement pourvu en azote $(0,2 \%$ ) et présente un rapport $\mathrm{C} / \mathrm{N}=13$; ce qui indique une lente minéralisation de la matière organique. Ses teneurs en phosphore disponible sont aussi faibles. Il y a un assez bon équilibre entre les teneurs en $\mathrm{K}, \mathrm{Mg}$ et $\mathrm{Ca}$, ce qui éloigne les risques d'antagonisme entre ces éléments, quant à leur absorption par la plante (Boyer, 1982). Ce sol est moyennement saturé en bases échangeables $(\mathrm{V}=65 \%)$ mais sa CEC est très faible. Il est très acide $(\mathrm{pH}=$ $4,45)$ pouvant passer à hyper acide $(\mathrm{pH}=$ 3,64 ) en profondeur au niveau de la haute vallée. Il est jugé non salé.

Les caractéristiques morphologiques et analytiques des différentes unités de sols rencontrées dans la vallée de Tamra sont résumées dans le Tableau 1 et 2.

Observons également, ci-dessous, la distribution des sols suivant la topovariance au niveau des 3 unités morpho-pédologiques identifiées le long de la vallée de Tamra (Figures 3, 4 et 5).

L'aptitude culturale proposée de ces sols a été appréciée sur la base de la nature du sol et le niveau topographique où on l'a rencontré. 
Tableau 1 : Résultats des analyses de sols et de nappes.

\begin{tabular}{|c|c|c|c|c|c|c|c|c|c|c|c|c|c|c|c|}
\hline \multicolumn{2}{|l|}{$\mathbf{N}^{\circ}$ échantillon } & G6008 & G6009 & G6015 & G6016 & G6017 & G6018 & G6019 & G6020 & G6021 & G6022 & G6023 & G6024 & G6025 & G6026 \\
\hline \multicolumn{2}{|l|}{$\mathbf{N}^{\circ}$ profil } & JBP 2 & JBP 2 & JBP 9 & JBP 9 & JBP 9 & JBP 9 & JBP 9 & JBP 12 & JBP 12 & JBP 15 & JBP 15 & JBP 15 & JBP 15 & JBP 15 \\
\hline \multicolumn{2}{|l|}{ Horizons $(\mathrm{cm})$} & $0-37$ & $78-140$ & $0-20$ & $20-50$ & $50-76$ & $76-102$ & $102-150$ & $0-47 / 50$ & $91-150$ & $0-20$ & $20-50$ & $50-76$ & $76-102$ & $102-150$ \\
\hline \multicolumn{2}{|l|}{$\mathrm{CE}(\mathrm{mS} / \mathrm{cm})$} & 10,529 & 12,421 & 0,852 & 0,313 & 0,591 & 1,183 & 2,898 & 0,044 & 0,26 & 0,227 & 0,225 & 0,134 & 0,101 & 0,304 \\
\hline \multicolumn{2}{|l|}{$\mathrm{pH}$ eau } & 3,52 & 2,4 & 4,32 & 3,90 & 3,56 & 3,46 & 2,63 & 4,45 & 3,91 & 3,89 & 3,59 & 3,6 & 3,83 & 4,68 \\
\hline \multicolumn{2}{|l|}{$\mathrm{pH} \mathrm{KCl}$} & 3,41 & 2,3 & 3,94 & 3,58 & 3,44 & 3,38 & 2,5 & 3,94 & 3,64 & 3,78 & 3,55 & 3,54 & 3,8 & 3,74 \\
\hline \multirow{3}{*}{$\begin{array}{l}\text { Granulométrie } \\
(\%)\end{array}$} & Argiles & 13 & 13,3 & 5,8 & 8,5 & 6,3 & 6,3 & 2,3 & 5,5 & 8 & 7,5 & 5,5 & 3,5 & 1,8 & 0,5 \\
\hline & Limons & 10,5 & 13,6 & 14,3 & 4,1 & 3,7 & 4,1 & 7,2 & 11 & 4,4 & 10,1 & 4,2 & 1,1 & 2,5 & 1,4 \\
\hline & Sables & 75,8 & 65 & 82 & 87 & 89,4 & 88,6 & 90,3 & 82,6 & 87,4 & 82,7 & 92,3 & 95,5 & 95,9 & 97,9 \\
\hline \multicolumn{2}{|l|}{ Carbone $(\% o)$} & & & 11,14 & 1,59 & & & & 2,59 & & 8,05 & 1,91 & & & \\
\hline \multicolumn{2}{|l|}{ Azote total $(\% o)$} & & & 0,89 & 0,17 & & & & 0,20 & & 0,53 & 0,14 & & & \\
\hline \multicolumn{2}{|l|}{$\mathrm{C} / \mathrm{N}$} & & & 12 & 9 & & & & 13 & & 15 & 14 & & & \\
\hline \multicolumn{2}{|c|}{$\mathrm{P}_{2} \mathrm{O}_{5}$ assimila. (ppm) } & & & 18 & 3 & & & & 3 & & 4 & 2 & & & \\
\hline \multirow{6}{*}{$\begin{array}{l}\text { Complexe } \\
\text { adsorbant (meq/ } \\
100 \mathrm{~g})\end{array}$} & $\mathrm{Ca}$ & & & 0,92 & 0,28 & & & & 0,2 & & 0,23 & 0,11 & & & \\
\hline & $\mathrm{Mg}$ & & & 1,12 & 0,43 & & & & 0,1 & & 0,16 & 0,11 & & & \\
\hline & $\mathrm{Na}$ & & & 2,17 & 0,75 & & & & 0,036 & & 0,205 & 0,199 & & & \\
\hline & $\mathrm{K}$ & & & 0,127 & 0,087 & & & & 0,052 & & 0,045 & 0,044 & & & \\
\hline & $\mathrm{S}$ & & & 4,34 & 1,55 & & & & 0,39 & & 0,64 & 0,68 & & & \\
\hline & $\mathrm{T}$ & & & 1,6 & 1,4 & & & & 0,6 & & 1,20 & 1 & & & \\
\hline
\end{tabular}


J. H. B. SENE et al. / Int. J. Biol. Chem. Sci. 8(2): 794-810, 2014

\begin{tabular}{|c|c|c|c|c|c|c|c|c|c|c|c|c|c|c|c|}
\hline $\mathbf{N}^{\circ}$ échantillon & & G6010 & G6011 & G6015 & G6016 & G6017 & G6018 & G6019 & G6022 & G6023 & G6024 & G6025 & G6026 & G6031 & G6032 \\
\hline $\mathbf{N}^{\circ}$ profil & & JBP 3 & JBP 3 & JBP 9 & JBP 9 & JBP 9 & JBP 9 & JBP 9 & JBP 15 & JBP 15 & JBP 15 & JBP 15 & JBP 15 & JBP 2 & JBP 9 \\
\hline Horizons $(\mathrm{cm})$ & & $0-20$ & $80-140$ & $0-20$ & $20-50$ & $50-76$ & $76-102$ & $102-150$ & $0-20$ & $20-50$ & $50-76$ & $76-102$ & $102-150$ & Nappe & Nappe \\
\hline $\mathrm{CE}(\mathrm{mS} / \mathrm{cm})$ & & 1,745 & 7,689 & 0,852 & 0,313 & 0,591 & 1,183 & 2,898 & 0,227 & 0,225 & 0,134 & 0,101 & 0,304 & 26 & 120 \\
\hline \multirow{5}{*}{ Anions (meq/L) } & $\mathrm{CO}_{3}{ }^{2-}$ & Traces & Traces & Traces & Traces & Traces & Traces & Traces & Traces & Traces & Traces & Traces & Traces & Traces & Traces \\
\hline & $\mathrm{HCO}_{3}^{-}$ & 0,20 & 0,10 & 0,10 & 0,10 & 0,10 & Traces & 0,10 & 0,10 & Traces & Traces & Traces & Traces & Traces & Traces \\
\hline & $\mathrm{Cl}^{-}$ & 63,10 & 4,93 & 1,97 & 4,01 & 4,44 & 7,44 & 0,77 & 0,63 & 0,56 & 0,56 & 1,48 & 225,5 & 943,7 & 943,7 \\
\hline & $\mathrm{SO}_{4}{ }^{2-}$ & 8,19 & 2,02 & 1,12 & 2,67 & 3,33 & 19,00 & 1,30 & 0,67 & 0,49 & 0,40 & 1,30 & 33,2 & 128,6 & 128,6 \\
\hline & Somme & 71,29 & 7,05 & 3,19 & 6,78 & 7,87 & 26,44 & 2,17 & 1,40 & 1,05 & 0,96 & 2,78 & 258,7 & 1072 & 1072 \\
\hline \multirow{5}{*}{ Cations (meq/L) } & $\mathrm{Ca}^{2+}$ & 3,00 & 0,50 & 0,20 & 0,30 & 0,70 & 1,15 & 0,40 & 0,11 & 0,10 & 0,05 & 0,15 & 15,0 & 60,0 & 60,0 \\
\hline & $\mathrm{Mg}^{2+}$ & 13,00 & 1,40 & 0,40 & 1,40 & 1,80 & 3,90 & 0,36 & 0,32 & 0,19 & 0,15 & 0,52 & 42,6 & 248,0 & 248,0 \\
\hline & $\mathrm{Na}^{+}$ & 53,20 & 4,60 & 2,80 & 2,50 & 6,50 & 11,80 & 0,90 & 0,90 & 0,50 & 0,35 & 1,46 & 144,5 & 752,0 & 752,0 \\
\hline & $\mathrm{K}^{+}$ & 1,13 & 0,15 & 0,08 & 0,08 & 0,20 & 0,48 & 0,04 & 0,05 & 0,03 & 0,03 & 0,03 & 4,0 & 20,0 & 20,0 \\
\hline & Somme & 70,33 & 6,65 & 3,48 & 4,28 & 9,20 & 17,33 & 1,70 & 1,38 & 0,82 & 0,58 & 2,16 & 206,1 & 1080 & 1080 \\
\hline
\end{tabular}

Tableau 2 : Synthèse sur les caractéristiques des différentes unités de sols.

\begin{tabular}{|c|c|c|c|c|c|}
\hline \multirow[t]{2}{*}{ caractères } & & \multicolumn{4}{|c|}{ Unités de sol } \\
\hline & & US 1 & US 2 & US 3 & US 4 \\
\hline \multirow{2}{*}{ Humidité } & Surface & Humide & $\mathrm{Sec}$ & $\mathrm{Sec}$ & $\mathrm{Sec}$ \\
\hline & Profondeur & Engorgé & Engorgé & Humide à engorgé & Humide à engorgé \\
\hline \multirow{2}{*}{ Couleur } & Surface & Gris clair & Gris clair & Gris foncé & Beige jaunâtre \\
\hline & Profondeur & Noir & Noir & Gris blanchâtre & Gris blanchâtre \\
\hline Taches & $\begin{array}{l}\text { Surface } \\
\text { Profondeur }\end{array}$ & $\begin{array}{c}\text { Bariolé ocre rougeâtre et jaunâtre à } 90 \% \\
\text { Plages ocres jaunes verdâtres }\end{array}$ & $\begin{array}{l}\text { Nombreuses taches ocres } \\
\text { Plages noires foncées }\end{array}$ & $\begin{array}{l}\text { Bariolé ocre jaunâtre } \\
\text { Plages ocres brunes }\end{array}$ & $\begin{array}{c}\text { Grossières taches rougeâtres } \\
\text { Bariolé ocre jaunâtre }\end{array}$ \\
\hline Texture & $\begin{array}{l}\text { Surface } \\
\text { Profondeur }\end{array}$ & $\begin{array}{l}\text { Sableuse } \\
\text { Sableuse }\end{array}$ & $\begin{array}{l}\text { Sableuse } \\
\text { Sableuse }\end{array}$ & $\begin{array}{l}\text { Sableuse } \\
\text { Sableuse }\end{array}$ & $\begin{array}{l}\text { Sableuse } \\
\text { Sableuse }\end{array}$ \\
\hline Structure & Surface & Continue & Polyédrique fine & Polyédrique fine & Polyédrique fine \\
\hline
\end{tabular}

801 
J. H. B. SENE et al. / Int. J. Biol. Chem. Sci. 8(2): 794-810, 2014

\begin{tabular}{|c|c|c|c|c|c|}
\hline \multirow{3}{*}{ Porosité } & Profondeur & Massive & Continue & Continue & Continue \\
\hline & Surface & Peu poreux & Poreux & Peux poreux & Poreux \\
\hline & Profondeur & Peu poreux & Poreux & Poreux & Peu poreux \\
\hline \multirow[b]{2}{*}{ Consistance } & Surface & Peu compact & Fragile & Compact & Compact \\
\hline & Profondeur & Peu compact & Peu compact & Compact & Compact \\
\hline Inclusions & Profondeur & Iron pipes & Néant & Néant & Néant \\
\hline \multicolumn{2}{|l|}{$\mathrm{C}(\%)$} & - & 1,114 & 0,8 & 0,259 \\
\hline \multicolumn{2}{|l|}{$\mathrm{Nt}(\%)$} & - & 0,089 & 0,053 & 0,02 \\
\hline \multicolumn{2}{|l|}{$\mathrm{C} / \mathrm{N}$} & - & 12 & 15 & 13 \\
\hline \multicolumn{2}{|c|}{$\mathrm{P}_{2} \mathrm{O}_{5}$ assimilable (ppm) } & & 18 & 4 & 3 \\
\hline \multicolumn{2}{|c|}{ Matières organiques (\%) } & - & 1,9 (bon) & 1,38 (faible) & 0,045 (très faible) \\
\hline \multirow{2}{*}{$\mathrm{pH}$ eau } & Surface & 3,5 (très acide) & 4,3 (très acide) & 3,89 (très acide) & 4,5 (très acide) \\
\hline & Profondeur & 2,4 (hyper acide) & 2,6 (hyper acide) & 4,68 (très acide) & 3,91 (très acide) \\
\hline \multirow{2}{*}{$\mathrm{CE}(\mathrm{mS} / \mathrm{cm})$} & Surface & 10,529 (extrêmement salé) & 0,852 (peu salé) & 0,227 (non salé) & 0,044 (non salé) \\
\hline & Profondeur & 12,421 (extrêmement salé) & 2,9 (très salé) & 0,304 (non salé) & 0,26 (non salé) \\
\hline \multirow[t]{2}{*}{ TSE (\%) } & Horizon 1 & - & 135,625 & 17,08 & 6 \\
\hline & Horizon 2 & - & 53,57 & 19,9 & - \\
\hline \multirow{2}{*}{\multicolumn{2}{|c|}{ CEC (meq/100 g) }} & - & 1,60 & 1,20 & 0,60 \\
\hline & & - & - & 53 & 65 \\
\hline \multicolumn{6}{|c|}{ 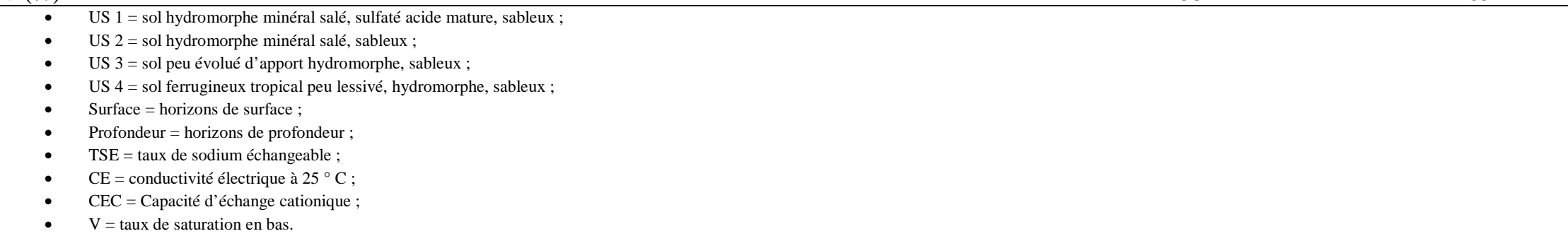 } \\
\hline
\end{tabular}




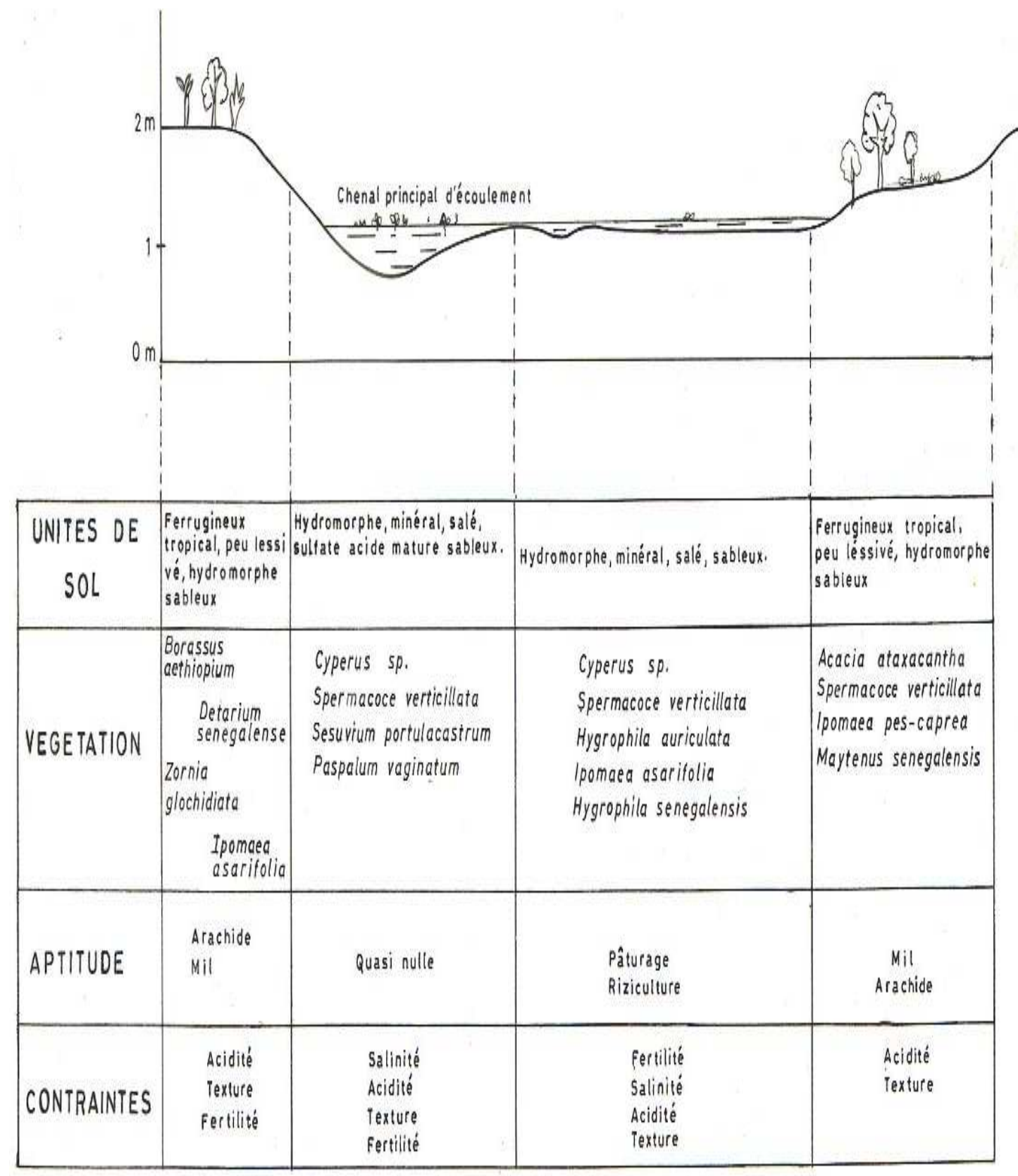

\section{DISTRIBUTION DES SOLS DANS LA BASSE VALLEE}

Figure 3 : Distribution des sols suivant la topovariance transversale dans la basse vallée. 


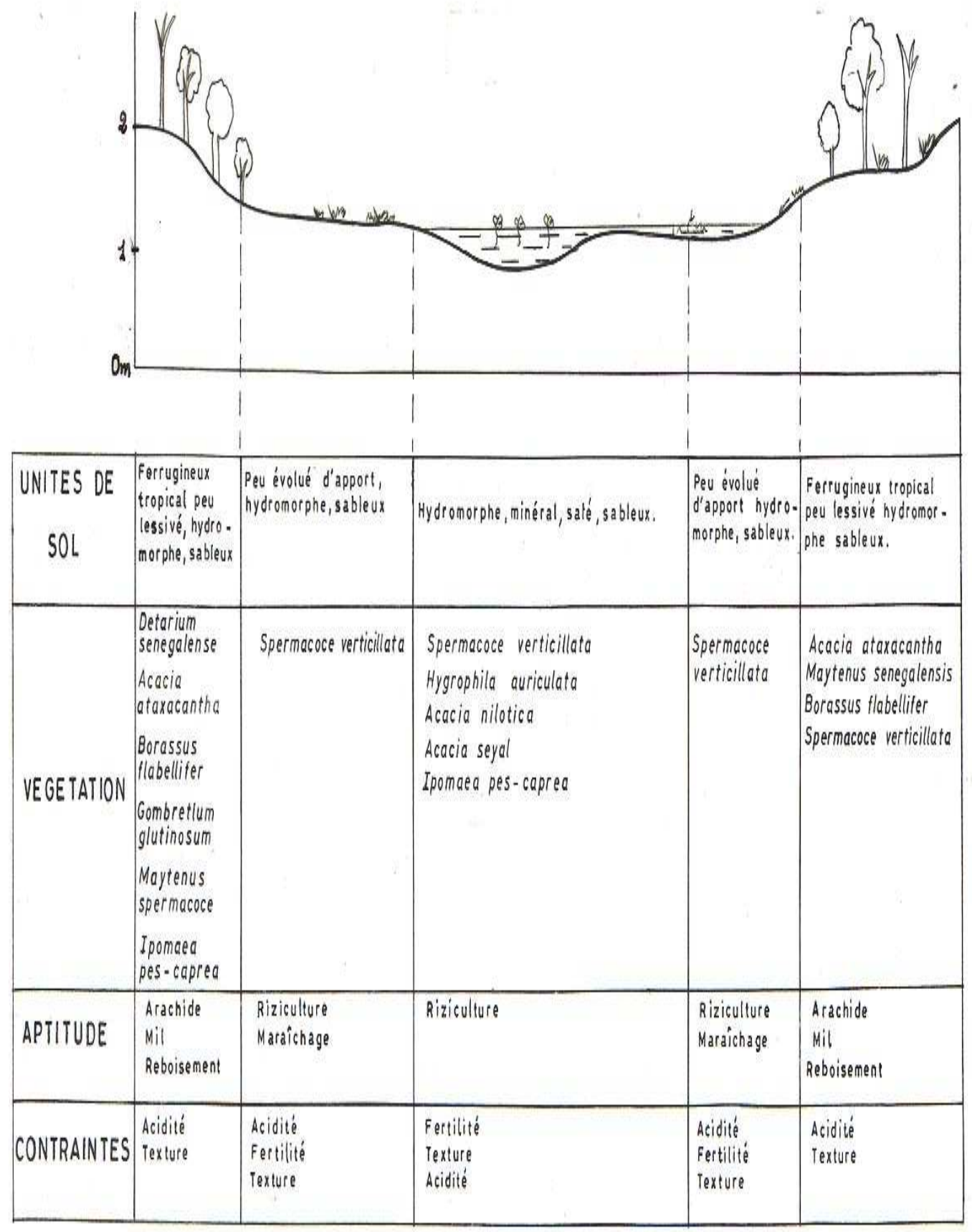

Figure 4 : Distribution des sols suivant la topovariance transversale dans la moyenne vallée. 
J. H. B. SENE et al. / Int. J. Biol. Chem. Sci. 8(2): 794-810, 2014



Figure 5 : Distribution des sols suivant la topovariance transversale dans la haute vallée. 


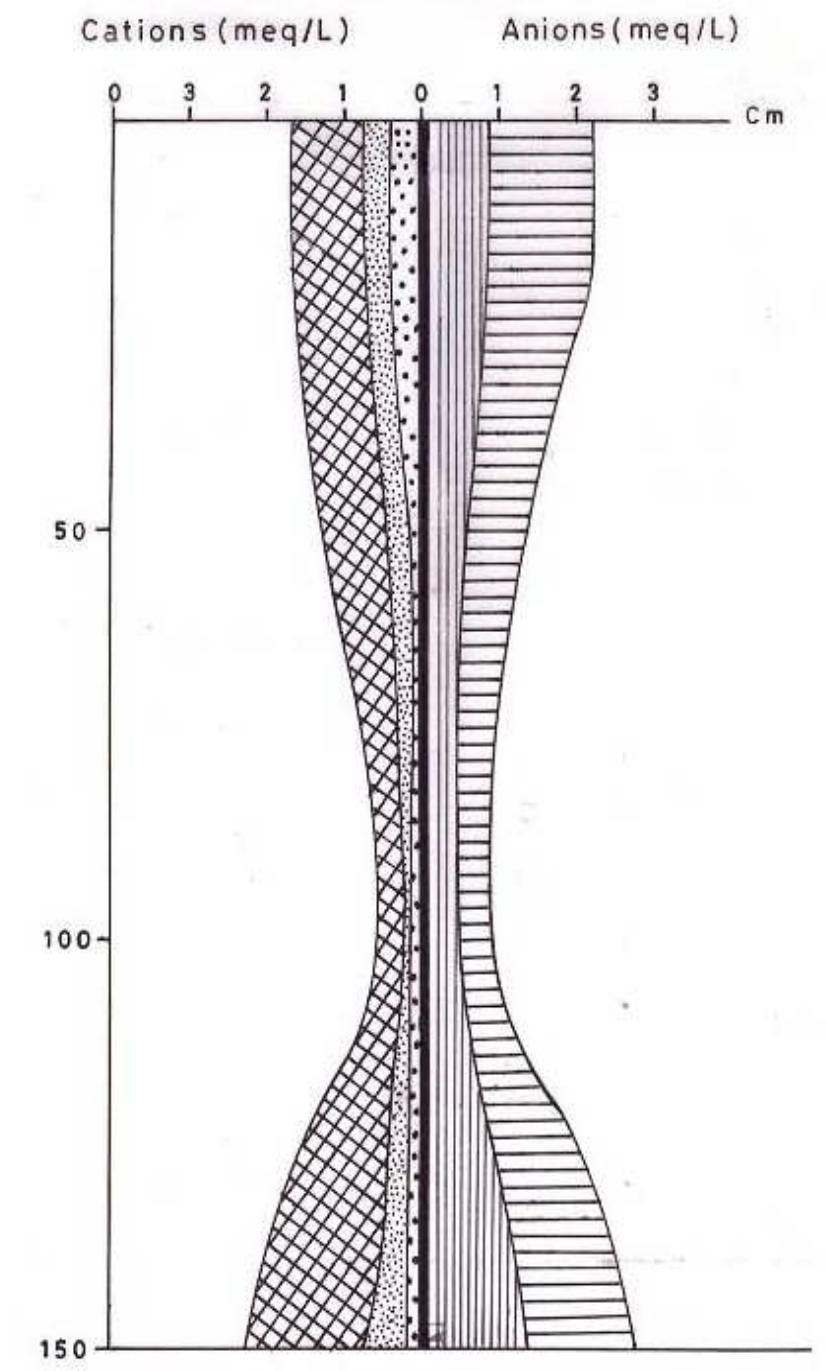

SOL PEU EVOLUE D'APPORT HYDROMORPHE SABLEUX (SABLES FINS)

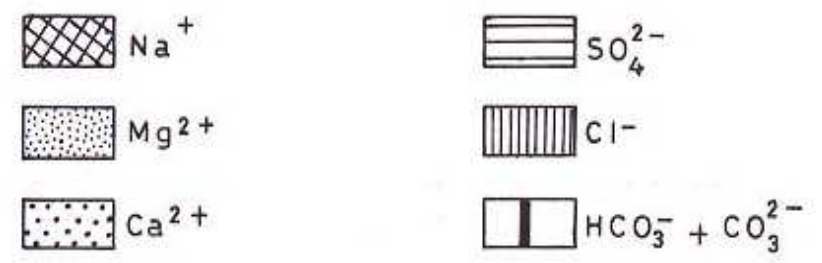

Figure 6 : Profil salin du sol au niveau de la haute vallée. 


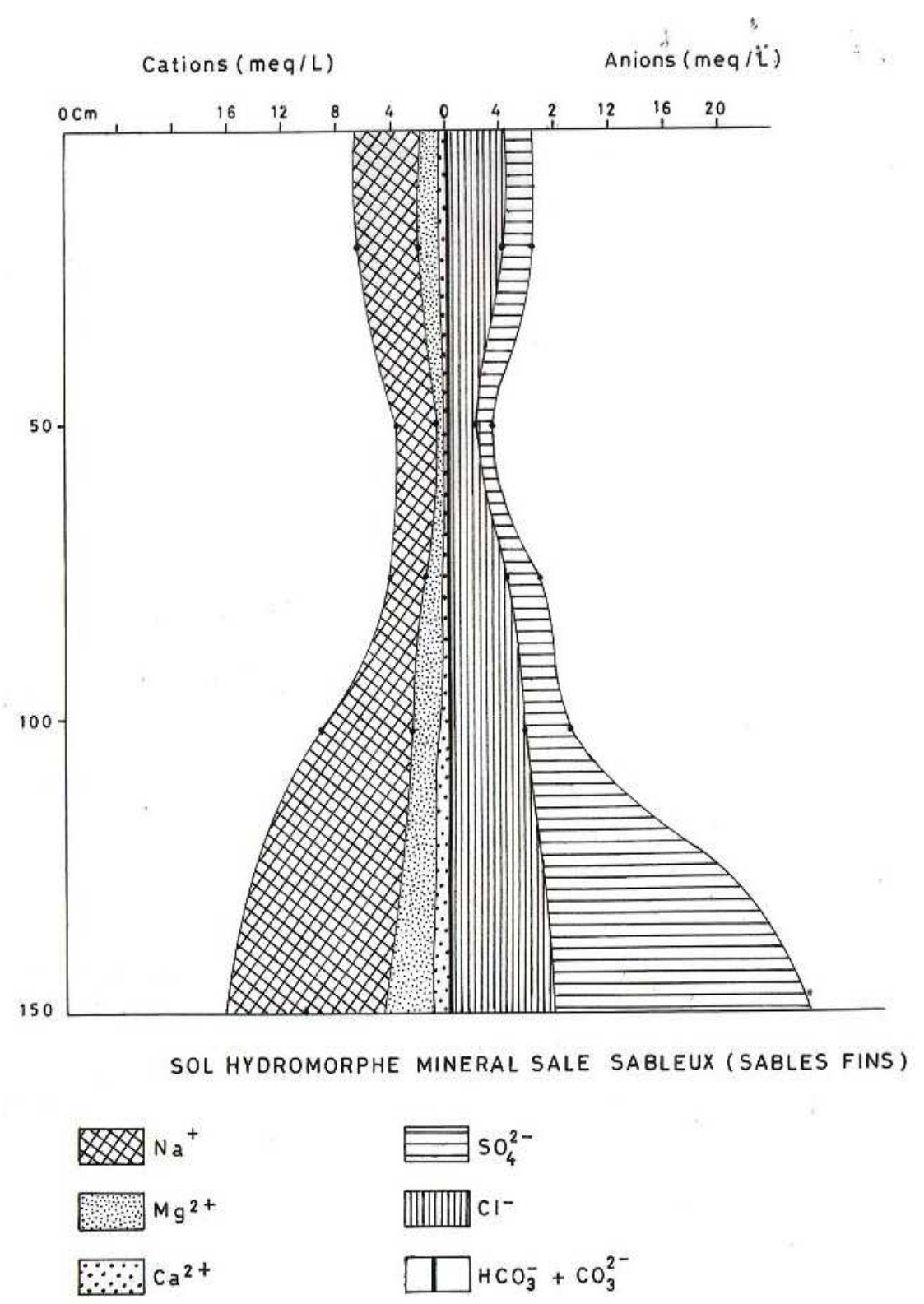

Figure 7 : Profil salin du sol au niveau de la moyenne vallée.

\section{DISCUSSION}

Très peu d'études pédologiques ont été menées dans le bassin fluvio-marin du SineSaloum notamment en zone insulaire où se situe l'île de Mar. Les rares études effectuées dans ce bassin-versant sont celles de Marius (1985) et Sadio (1991). Celles-ci se sont intéressées à la cartographie des sols à l'échelle globale d'où l'intérêt de la présente étude qui porte sur une échelle plus locale couvrant la vallée de Tamra pour mieux affiner la caractérisation des sols en vue de leur utilisation rationnelle par les populations. Elle est d'autant plus importante que la superficie des terres cultivables est de plus en plus réduite dans l'île alors que la population ne cesse d'augmenter. Les résultats discutés 807 
d'investigation, voire au niveau du périmètre rizicole.

Cette étude des sols menée à travers des séquences transversales et représentatives (Figures 3,4,5) révèle globalement qu'en terrasse basse, on rencontre principalement le sol hydromorphe, minéral salé, sulfaté acide et le sol hydromorphe minéral salé. La terrasse moyenne est occupée essentiellement par le sol peu évolué d'apport, hydromorphe. Enfin, en terrasse haute, on trouve le sol ferrugineux tropical, peu lessivé, hydromorphe. Ces différentes unités de sols identifiées sont disposées d'une manière quasi-concentrique autour de la vallée de Tamra. Ce caractère concentrique de la distribution des sols est quelque peu perturbé en haute vallée où l'on a constaté que le sol hydromorphe minéral salé, sulfaté acide et le sol hydromorphe minéral salé ont disparu. Cette disparition pourrait s'expliquer par le fait que l'influence marine devient de plus en plus faible sur le processus de formation des sols au fur et à mesure qu'on remonte la vallée. Rappelons que la vallée de Tamra est un affluent du marigot de Djilor, un cours d'eau sursalée, et qu'en haute vallée, les eaux douces de pluies semblent jouer un rôle prépondérant dans la pédogenèse. Ce rôle important des eaux de pluies a été renforcé par la présence des deux digues mentionnées ci-dessus (Figure 2). La première, située en basse vallée, joue le rôle de digue anti-sel et la deuxième est une digue de retenue. Les différents types de sols inventoriés doivent donc être appréciés en fonction des exigences de la culture du riz. Ces sols ont dans l'ensemble une texture sableuse, une capacité d'échange cationique faible et un taux de matière organique faible à moyen.

Notons qu'avec une texture aussi légère, les sols ne peuvent accumuler un stock de matière organique appréciable pouvant améliorer leur capacité d'échange cationique. Ils sont acides, salés notamment en basse et moyenne vallée. Leurs contraintes majeures, en rapport avec la riziculture, sont la texture légère du substrat, l'acidification et la salinisation.

\section{La texture légère des sols (sableuse)}

Le processus de formation des sols dans ce milieu insulaire est complexe. Les sables, matériaux constitutifs essentiels de ces sols, sont d'origine marine et éolienne (Sadio, 1991 ; Diarra, 1999). Le phénomène éolien est encore actif de nos jours, si bien qu'on assiste actuellement à un ensablement de la vallée. Il est à souligner qu'une texture lourde (argileuse, argilo-sableuse) assure le mieux la croissance et le développement du riz car elle permet au sol de retenir assez d'eau pour une alimentation correcte de la culture. Il est connu qu'un matériau sableux a une faible capacité de rétention en eau (Durand, 1983). Précisons que la riziculture pratiquée dans la vallée de Tamra est de type pluvial par submersion, d'où le caractère contraignant de la texture sableuse des sols.

\section{L'acidification des sols}

L'ensemble des unités de sols identifiées montrent des valeurs de $\mathrm{pH}$ acide à hyper-acide, notamment dans les horizons de profondeur. Cette acidité serait, entre autres facteurs, d'origine géologique dans ce milieu humide (Vieillefon, 1974). En effet, le matériau sablo-vaseux noirâtre trouvé en profondeur serait riche en pyrite $\left(\mathrm{FeS}_{2}\right)$ (Sadio, 1991). Exposé à l'air libre, il s'oxyde et donne de la jarosite $\left[\mathrm{KFe}_{3}(\mathrm{SO} 4)_{2}(\mathrm{OH})_{6}\right]$. Ce sel complexe, en se dissociant, produit dans le milieu de l'acide sulfurique $\mathrm{H}_{2} \mathrm{SO}_{4}$ (Van Breemen, 1987). Les valeurs de $\mathrm{pH}<5$ limitent la croissance et le développement de la culture du riz (Boyer, 1982 ; Brady et Weil, 2008). Cette acidité peut être combattue par le chaulage des sols, un amendement organique substantiel (fumier, compost) et la sélection de variétés de riz adaptées (Brady et Weil, 2008). En outre, il faut également veiller à une application rationnelle des engrais minéraux étant reconnu que certains d'entre eux ont un pouvoir acidifiant du sol. 


\section{La salinisation des sols}

Les données analytiques obtenues montrent qu'il existe un gradient de salinité décroissant de l'aval vers l'amont de la vallée et un gradient de salinité croissant de la surface vers la profondeur du sol. Ce gradient de salinité est perceptible à travers la forme des profils salins du sol dressés en moyenne et haute vallée (Figures 6 et 7). En effet, on note de fortes concentrations de sels solubles dans les horizons de profondeur par rapport aux horizons de surface. La texture de ces sols étant sableuse, elle favorise un lessivage vertical des sels solubles. L'identification du niveau de profondeur de la couche du sol la plus salée est d'une grande importance dans l'exploitation rationnelle des sols cultivés affectés par le phénomène de salinisation (Alexandrova et Naïdionova, 1976). Cela permet de faire un choix approprié quant aux spéculations à développer sur ces terres, d'où l'importance d'une bonne analyse de leurs profils salins. La salinité dans ce milieu fluvio-marin pourrait être combattue par l'apport du gypse et du phosphogypse, par un amendement organique, un drainage approprié des sols et par un entretien adéquat des digues existantes afin de favoriser le stockage des eaux douces de pluies.

\section{Conclusion}

La couverture pédologique de la vallée de Tamra est complexe. Quatre unités de sols sont identifiées. Elles sont imbriquées les unes aux autres suivant une disposition quasiconcentrique autour de la vallée. La salinité et l'acidité constituent les principales contraintes à la production agricole dans cette vallée rizicole de Tamra. La nappe phréatique est très salée voire hyper-salée $(\mathrm{CE}=26$ à 120 $\mathrm{mS} / \mathrm{cm}$ ) et les sels remontent par capillarité à la surface du sol. Les périodes récurrentes de sécheresse qui sévissent ces 30 dernières années au Sénégal ont fortement favorisé cette salinisation des terres. Ainsi, la dilution de cette nappe d'eau salée par les eaux de pluies serait une des solutions efficaces pour dessaler ces sols. Le renforcement du rôle des deux digues existantes contribuerait à améliorer le dessalement de ces sols d'autant plus que leur texture est sableuse; ce qui est un atout majeur pour le lessivage des sels. La texture sableuse mais fine permet, quelque peu, de retenir de l'eau pour la riziculture dans cette vallée. Cependant, le caractère très exondé de certaines unités de sols rend indispensable la réalisation d'une carte d'aptitude culturale des sols pour une exploitation rationnelle des terres de la vallée de Tamra, très sollicitée par les populations des quatre villages de l'île de Mar. En effet, le manque de terres cultivables dans l'île et la croissance démographique font que même les terres marginales de la vallée sont exploitées par les populations pour la production du riz. Une diversification des cultures pourrait être envisagée pour une exploitation rationnelle de ces terres.

\section{REFERENCES}

Alexandrova LN, Naïdionova OA. 1976. Interprétation des résultats d'analyse de l'extrait 1/5 du sol. In Manuel Pratique de Pédologie, "Kolos" (3 ${ }^{\text {ème }}$ édn). Léningrad : 176-184.

Baize D. 1988. Guide des Analyses Courantes en Pédologie. INRA: Paris; 172 p.

Barusseau JP, Diop ES, Saos JL. 1985. Evidence of dynamics reversal in tropical estuaries, geomorphological and sedimentological consequences (Salum and Casamance Rivers, Senegal). Sedimentology, 32: 543-552.

Black CA, Evan DD. 1965. Methods of soil analysis. American Society of Agronomy, Part 2, Agronomy, 9: 917-918.

Boyer J. 1982. Les Sols Ferralitiques: Facteurs de Fertilité et Utilisation des Sols (Tome X). Collection InitiationsDocumentations Techniques de l'Orstom: Paris; 384 p.

Brady NC, Weil RR. 2008. The Nature and Properties of Soils $\left(14^{\text {th }}\right.$ edn). Pearson Education Inc.: New York; 1046 p.

Bray RH, Kurtz LT. 1945. Determination of total, organic and available forms of 
phosphorus in soils. Soil Science, 59: 3945.

CPCS. 1967. Classification des Sols. Ecole Nationale de Sciences Agronomiques, CPCS: Paris ; 67 p.

Diarra M. 1999. Formation et évolution finiholocènes et dynamique actuelle du delta Saloum-Gambie (Sénégal-Afrique de l'ouest): géomorphologie, stratigraphie, sédimentologie et dynamique sédimentaire. Thèse de Doctorat, Université de Perpignan (FRAN), 159 p.

Diop ES. 1986. Estuaires holocènes tropicaux. Etude de géographie physique comparée des « rivières du sud»: du Saloum (Sénégal) à la Mellacorée (République de Guinée). Thèse de Doctorat d'Etat, Université de Strasbourg, 565 p.

Durand JH. 1983. Les sols Irrigables. Agence de Coopération Culturelle et TechniqueConseil International de la Langue Française: Paris; 339 p.

Marius C. 1979. Effets de la sécheresse sur l'évolution phytogéographique et pédologique de la mangrove en basse Casamance. Bulletin de l'IFAN, Série A :
Sciences Naturelles, Dakar, 41(4) : 669691.

Marius C. 1985. Mangrove du Sénégal et de la Gambie : écologie, pédologie, géochimie, mise en valeur et aménagement. Thèse Doctorat ès Sciences Naturelles, Université Louis Pasteur de Strasbourg, $335 \mathrm{p}$.

Sadio S. 1991. Pédogenèse et potentialités forestières des sols sulfatés acides salés des tannes du Sine-Saloum (Sénégal). Thèse d'état, Orstom éditions, Dakar, 269 p.

Van Breemen N. 1987. Effects of redox processes on soil acidity. Netherlands Journal of Agricultural Sciences, Wageningen, 35: 271-279.

Vieillefon J. 1974. Quelques conséquences des transformations du soufre sur la pédogenèse dans une séquence de sols du domaine fluvio-marin tropical. Cahiers Orstom, série Pédologie, Dakar, 12(1): 47-60. 